\title{
SYLTEC : un procédé innovant pour le recyclage des matériaux composites à matrice thermodurcissable
}

\author{
D. Perrin, E. Leroy , L. Clerc, A. Bergeret et J.M. Lopez-Cuesta \\ École des Mines, CMGD, 6 avenue de clavières, 30319 Alès Cedex, France
}

\author{
Mots-clés : \\ Recyclage; valorisation; SMC; \\ Sheet Moulding Compound; \\ composites; polypropylène; \\ interface; procédé; SYLTEC; \\ broyage; dissolution; carbonate de \\ calcium; fibre de verre; polyester; \\ traitement thermique; DETA; \\ traitement chimique; propriétés \\ mécaniques
}

\begin{abstract}
Résumé - L'objectif de l'étude est d'améliorer les propriétés mécaniques de matériaux composites SMC recyclés incorporés dans une matrice polypropylène. Pour cela, nous avons proposé un procédé de valorisation de ces charges SMC, dénommé SYLTEC, en quatre étapes: La première étape, phase de conditionnement, a eu pour objet une libération des fibres de verre de la résine polyester ainsi qu'une calibration dimensionnelle du broyat de SMC sous forme de matière plus ou moins cotonneuse. L'utilisation d'un déchiquetage a permis une préservation de la longueur des fibres tout en conservant un taux des constituants stable, et plus particulièrement, une teneur fibreuse qui se maintient lors de ce broyage primaire. La deuxième étape, phase de renforcement matériau, consiste en une étape de dissolution contrôlée du carbonate de calcium par l'action d'une réaction acide-base et a permis, par le biais d'un ajustement du taux de fibres de verre, d'obtenir une fraction renforçante de 40-45\% en masse.

La troisième étape, phase de traitement thermique, a créé, par fonctionnalisation du polyester, une adhésion ionique, charge/charge (carbonate de calcium - anhydride du polyester thermiquement activé) et charge/matrice (carbonate de calcium - anhydride de l'agent interfacial $\mathrm{PP}_{\text {gma }}$ ). Cela a permis un renforcement conséquent du composite SMC/matrice PP permettant d'augmenter l'ensemble des propriétés mécaniques.

La dernière étape, phase de traitement chimique, a apporté une fonctionnalisation chimique entre la charge (SMC modifiée traitée thermiquement) et la matrice active (PP/PP gma $_{\text {) }}$ par l'intermédiaire d'un agent polyfonctionnel type amine. La condensation en imide a permis ainsi la création d'une interface chimique alors qu'elle était ionique après la troisième étape. Cette étape n'est pas nécessaire dans le cas d'une matrice polaire comme le PA-6.

Au bilan, nous sommes arrivés à obtenir, par le biais du procédé de valorisation de charges SMC recyclés incorporées dans du polypropylène, des propriétés mécaniques sensiblement comparables à celles de fibres neuves incorporées dans la même matrice.
\end{abstract}

\section{Key words:}

Recycling; sheet moulding compound; composites; polypropylene; polyamide; interface; SYLTEC; mechanical properties

\begin{abstract}
Management of SMC plastic waste by the process of recycling SYLTEC. The objective of this study was to improve the mechanical properties of SMC composite waste, recycled as fillers in a polypropylene matrix. A four stages process, called SYLTEC, was developed.

The first step called conditioning step, aimed to extract glass fibres from the polyester resin and to obtain a cotton-like aspect for the ground material. The use of a shredding process allowed either to preserve a minimum fibre length or to maintain a constant rate of each SMC component, particularly glass fibres.

The second step called reinforcement step, consisted in a controlled dissolution step of calcium carbonate by an acido-basic reaction and allowed to obtain a reinforcing glass fraction of $40-45 \%$ wt.

The third step, called thermal treatment step, created an ionic adhesion, filler/filler (calcium carbonate-anhydride of thermally activated polyester) by polyester fonctionnalization and filler/matrix (calcium carbonate-anhydride of interfacial agent $\mathrm{PP}_{\text {gma }}$ blended with PP). This entailed a significant reinforcement of composite and a strong improvement of mechanical properties.

The last step, called chemical treatment step, lead in the case of PP, to a chemical fonctionnalization between the SMC filler, thermally modified and the polymer matrix made of a $\mathrm{PP}_{\mathrm{PP}}$ gma blend, caused by an amine type polyfonctionnal agent. Condensation into imide allowed the creation of a chemical interface in comparison with the ionic one resulting from the third step. It was also noticed that this last step was unnecessary in the case of a polar polymer host matrix such as PA6 to achieve high mechanical performance.

Finally, the whole process lead to very close mechanical properties for the reinforced thermoplastics to the same polymer reinforced with virgin fibres.
\end{abstract}


e souci de la préservation de l'environnement est une préoccupation croissante qui ne s'est généralisée que récemment. Peu à peu, ce sont tous les secteurs de l'activité humaine qui sont soumis à une critique de la part de chercheurs scientifiques, de citoyens, d'associations ou d'élus. Pour de nombreuses personnes, la chimie et le pétrole sont souvent connotés négativement en ce qui concerne les impacts sur les écosystèmes et sur la santé. Les matières plastiques et composites issues de la pétrochimie risquent donc elles aussi d'être suspectes aux yeux de nos concitoyens. Ceci est renforcé par le fait que le développement de l'utilisation de ces matières est relativement récent et que, pour certains, les plastiques et composites demeurent des produits de substitution moins chers mais moins nobles. La pollution visuelle qui leur est due est souvent très mal perçue. Il est difficile de comprendre pourquoi les plastiques ne sont pas entièrement recyclés comme le verre, les métaux ou même le papier-carton. Régulièrement des campagnes de presse remettent en cause tel plastique ou telle utilisation ou s'inquiètent de la fiabilité des techniques d'élimination des déchets.

Pour ces raisons, il est légitime de se poser la question des rapports entre plastiques et environnement. C'est le but de cette étude, appliquée à une famille de matériaux plastiques dits armés : les composites à matrice polyester renforcés fibres de verre et carbonate de calcium (SMC).

Très peu de publications font état de recyclage mécanique ou physique des SMC, de même le nombre d'entreprises impliquées dans les activités industrielles correspondantes est également très faible. Aussi, un enjeu scientifique, technologique et économique est réellement présent pour ce mode de recyclage en apportant des réponses en terme de procédé rentable et performant visà-vis des divers matériaux composites à recycler.

L'objectif de ce projet est de concevoir et de développer un procédé de recyclage des composites SMC par incorporation au sein de matrices thermoplastiques capable de concurrencer, directement et sans substitution partielle, les propriétés générales des composites à fibres de verre vierges. Ce procédé Syltec de revalorisation du SMC aura pour but, par des modifications chimiques et thermiques, de fonctionnaliser de façon optimale, l'ensemble des matériaux constituant le composite final, afin d'améliorer l'adhésion entre chaque constituant.

Un thermoplastique apolaire de grande diffusion a été sélectionné pour l'étude : le polypropylène $(\mathrm{PP})$, en raison de son énorme tonnage annuel et parce qu'il est l'un des plus employés des composites à fibres de verre courtes. Nous avons également effectué, à titre de validation du procédé, une étude avec une matrice polaire, le PA-6, largement utilisée dans le monde industriel en combinaison avec des fibres de verre (non mentionné dans ce rapport).

Tout au long de cette étude, nous nous sommes efforcés de garder à l'esprit que la performance d'un procédé innovant de recyclage se doit d'apporter des solutions à la fois pragmatiques et peu onéreuses en terme de développement durable. Ceci constitue le cahier des charges que nous nous sommes fixé afin de garantir les performances mécaniques d'un matériau composite à charges recyclées SMC compétitif vis-à-vis de composites à fibres neuves.

Le procédé de valorisation de ces charges SMC se compose de quatre étapes :

- conditionnement,

- renforcement matériau,

- traitement thermique,

- traitement chimique dans le cas de matrice hôte polaire.

\section{Contexte}

\section{Le composite SMC}

Le SMC (Sheet Moulding Compound, matériau composite à base de fibres de verre, de carbonate de calcium et de résine polyester) représente un allégement et une alternative de faible coût par rapport aux métaux, permettant de réduire de $40 \%$ la masse de l'automobile. Il constitue désormais un matériau de choix dans plusieurs applications (automobiles comme les pièces de carrosseries) [1-3].

Les SMC représentent aujourd'hui environ $30 \%$ de la production annuelle des composites en Europe, soit environ 300 kt dont $95 \mathrm{kt}$ en France.

De par leur légèreté, leur longévité, leurs propriétés anti-corrosion, leur faisabilité de mise en œuvre, les matériaux composites SMC présentent un énorme potentiel de développement que ce soit dans l'automobile, les transports, l'industrie électrique et électronique, l'aéronautique, le bâtiment et l'habitat, le médical ou le sport. 
Cependant, bien que le marché des matériaux composites soit en plein essor (1,6 Mt en Europe, soit $1 / 4$ du marché mondial), dont $65 \%$ à base de thermodurcissables $(270 \mathrm{kt}$ en France) [4], deux facteurs limitent le développement de ces matériaux : leur coût et leur cycle de vie. La maîtrise du devenir des composites à matrice thermodurcissable devient donc un enjeu majeur en relation avec leur développement.

À l'heure actuelle, la voie habituelle pour le traitement des composites en fin de vie est le stockage en Centre d'Enfouissement Technique (CET). Ainsi, plus de $90 \%$ des déchets de production en composites thermodurcissables sont actuellement mis en décharge, avec un coût moyen en forte augmentation (de $60 € / t$ en 2001 à $100 € / t$ en 2003) [5]. Le problème de fin de vie concerne l'ensemble de la filière, du producteur de matrices ou de renforts jusqu'aux donneurs d'ordres en passant par des transformateurs. Cependant, certaines solutions de valorisation existent afin de proposer une alternative aux CET.

\section{Les voies de valorisation des composites SMC}

\section{Problématique}

Les matériaux composites SMC sont en progression dans tous les domaines d'application du fait de leurs bonnes caractéristiques techniques, de leur facilité de mise en œuvre et de leur faible coût de fabrication. Cependant, la nature chimique de la structure thermodurcissable les rend infusibles et non solubles contrairement aux thermoplastiques, et donc, n'autorise pas a priori la possibilité de les recycler. Les compoundeurs (MCR, ERCOM, VALCOR, SMC Automotive Alliance, etc.) se sont mobilisés pour proposer des solutions concrètes, et ce, sous la pression des gouvernements promulguant un durcissement de la législation et des directives dans les années à venir.

\section{Législations et directives}

En effet, l'existence de textes législatifs et réglementaires se rapportant à la gestion des déchets constitue l'élément essentiel vis-àvis de la gestion des composites en fin de vie.

Les objectifs annoncés par exemple pour les VHU sont respectivement de $80 \%$ de recyclage et réutilisation et de $85 \%$ de valorisation totale en 2006 , et de $85 \%$ et $95 \%$ en 2015. L'ensemble des constructeurs pense que, dans quelques années et au prix de quelques améliorations, la filière de recyclage des VHU pourrait devenir tout à fait rentable et créer des emplois [6].

\section{Contexte général}

Dans ce contexte, les grands pôles mondiaux de production de matériaux composites cherchent actuellement des solutions pour valoriser les matériaux en fin de vie. Du fait de réglementations propres et de cultures différentes, les solutions envisagées diffèrent mais la problématique reste la même. Dans ce cadre, de nombreux programmes et études ont été menés depuis le début des années 90 pour trouver des voies de valorisation matière, solvolyse, pyrolyse, combustion, etc. [7]. Ainsi, différentes techniques de valorisation ont été développées ou explorées ces dernières années. L'ensemble des traitements et valorisations actuels est synthétisé figure 1 .

Dans le cadre de l'étude, nous nous intéresserons plus précisément à la valorisation matière des composites SMC en tant que renforts au sein de thermoplastiques (TP).

\section{La valorisation matière des composites SMC}

\section{Techniques d'élaboration et matières recyclées SMC obtenues}

Cette valorisation permet de réduire les composites en deux étapes : déchiquetage (en morceaux de quelques centimètres carrés) et broyage.

La valorisation matière par broyage consiste à broyer des déchets pour les incorporer dans une nouvelle structure composite. Dans la plupart des cas, la démarche de broyage comprend le déchiquetage initial du composite en morceaux plus petits puis le broyage de ces morceaux en une poudre très fine qui peut être réutilisée comme charge active en remplacement des charges commerciales standard.

L'équipement standard pour le broyage des thermodurcissables peut comprendre [11] (Fig. 2) :

- un broyeur primaire tel un broyeur à marteaux, 


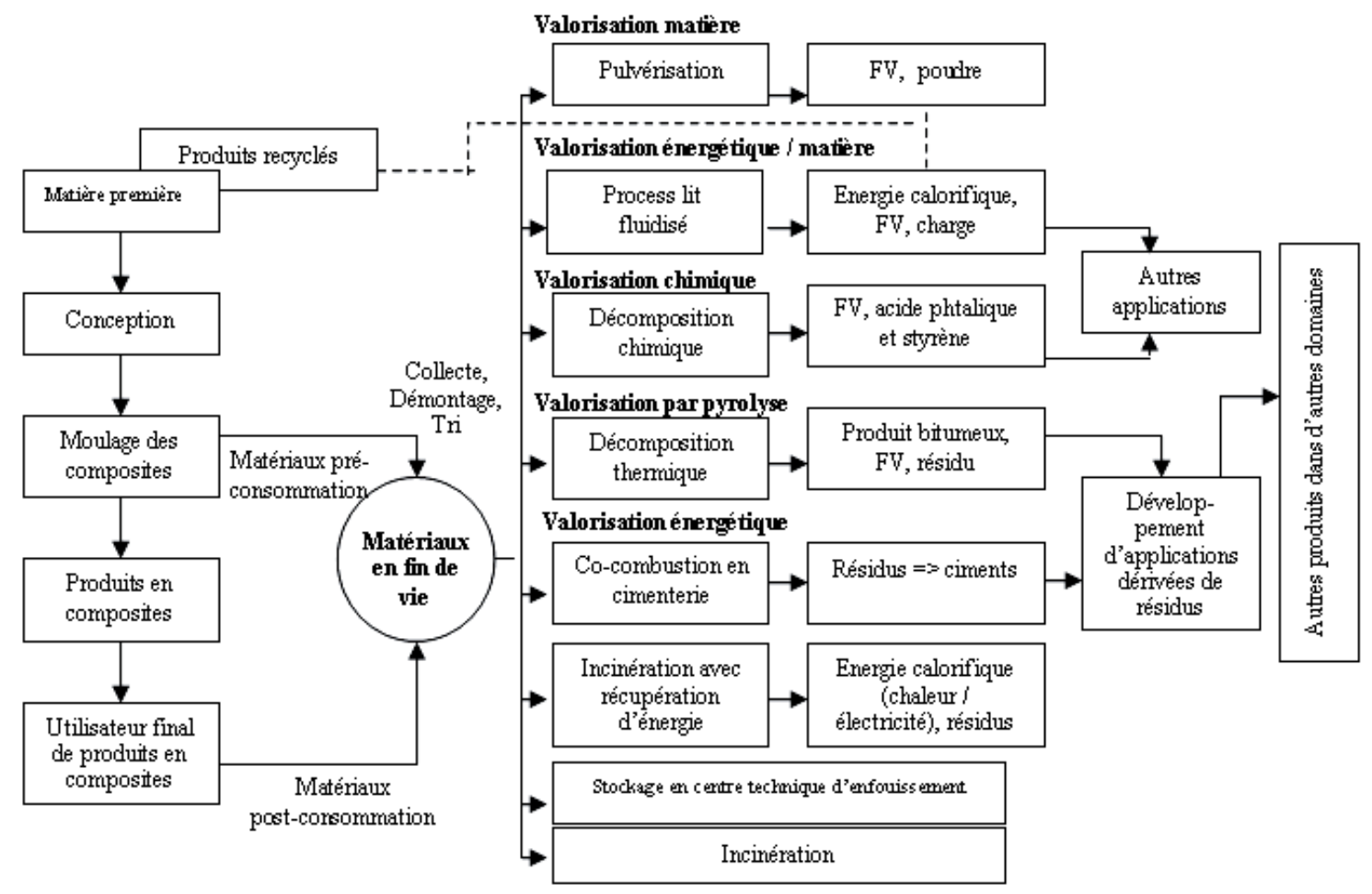

Fig. 1. Différentes techniques de traitement et valorisation des matériaux composites [5].

Fig. 1. Various techniques of treatment and management of composite material waste [5].

- un pulvérisateur pour transformer les matériaux en poudre fine,

- un séparateur pour séparer les différentes fractions.

En fonction du type de broyeur et de l'importance du broyage, le matériau valorisé peut être classé en 2 catégories [11] :

- une fraction fibreuse contenant la plus grande portion d'agents renforçants (fibres),

- une fraction de poudre fine contenant la plus grande partie de la matrice polymère.

Un procédé breveté par la société Phoenix Fiberglass permet ainsi de récupérer les fibres tandis que les déchets restants sont micronisés sous forme de poudres [12].

La société Seawolf Industries Inc. a également développé un système de broyage permettant de récupérer les fibres sans trop dégrader les fibres, celles-ci sont utilisables comme renfort de composite plutôt qu'en tant que charge [12]. Le matériau broyé est mélangé avec une mousse synthétique à base de polyester qui peut être pulvérisée. Baptisé Scrap Recycling System (brevet US $5,036,788$ ), ce système permettrait la production d'articles tels que les baignoires ou les ponts de bateaux. Ce débouché couvrirait rapidement son coût en éliminant les déchets solides et en réduisant l'utilisation de résine et de fibres de verre vierges.

La seule unité de recyclage français a été créée au sein de l'association Valcor. Cette association avait été mise en place sous l'impulsion de la société Mecelec Composites et Recyclage (devenue Mixte Composites Recyclage - MCR) qui, dès 1993, s'est intéressée à la maîtrise de la durée de vie des matériaux composites thermodurcissables (et en particulier les matériaux SMC/BMC), depuis la définition de la matière jusqu'à son recyclage complet, en passant par le développement, la validation, la production, l'utilisation et le traitement de fin de vie $[13,14]$.

En Allemagne, la première unité de traitement ayant vu le jour à Rastatt en 1991 a été la société ERCOM Composites Recycling $\mathrm{GmbH}$ [15]. Les principaux constructeurs automobiles (BMW, Mercedes) et les grands producteurs de SMC (Menzolit Fibron, Miltras...) ont créé ERCOM en association avec les principaux producteurs de matière première (St Gobain Vetrotex, Cray Valley. .. ).

L'unité d'une capacité de 5000 t/an est largement sous-employée. Seule la matière qui peut être vendue est traitée. Les déchets sont collectés et concassés sur les sites utilisateurs 


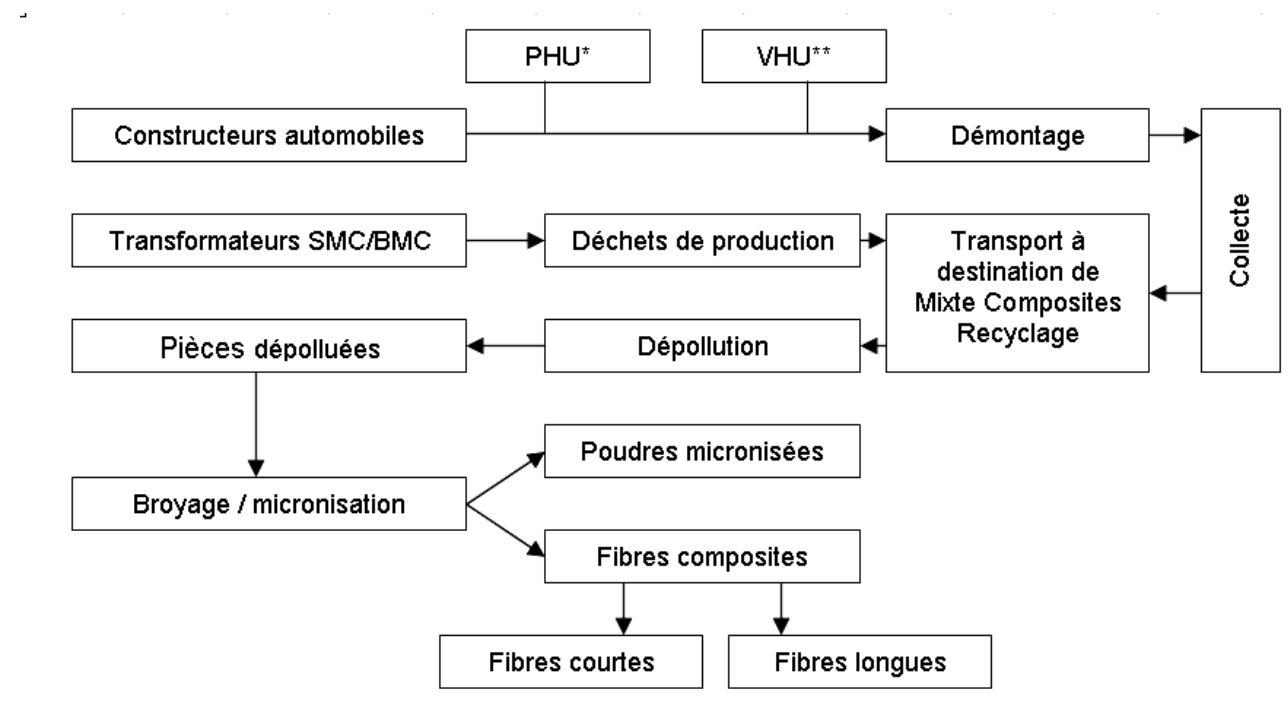

* PHU : Pièce hors d'usage

**VHU : véhicule hors d'usage

Fig. 2. Processus de recyclage des SMC/BMC chez MCR [11].

Fig. 2. Recycling process of SMC/BMC materials by MCR company [11].

de plastiques renforcés, les stations de démontage et les centres de ramassage, à l'aide d'une unité mobile de concassage.

\section{Débouchés}

Les débouchés pour la poudre micronisée et les fibres sont différents :

- La poudre micronisée de composites à matrice polyester peut être réintroduite comme charge en quantité contrôlée dans des formulations pour, entre autres, remplacer la résine qui coûte cher. Les structures sont ainsi plus légères. Cependant, l'utilisation de ces formulations nécessite une étude approfondie portant sur la formulation et sur le procédé. En effet, l'incorporation de la poudre recyclée entraîne une variation de la viscosité et requiert un ajustement entre les proportions de résine et de charges. Le nouveau mélange peut devenir alors plus cher. De plus, les caractéristiques mécaniques des mélanges formulés à base de poudre et de fibres de verre recyclées peuvent être plus faibles que celles du matériau vierge (sans traitements spécifiques). L'utilisation en boucle fermée n'est donc pas envisageable pour les pièces à caractéristiques mécaniques élevées. Il faut donc trouver des applications en boucle ouverte où les caractéristiques mécaniques recherchées ne sont pas prépondérantes (pièces d'aspect). Il existe de nombreuses possibilités d'emploi : coffrets électriques, réflecteurs, table de décoration, etc. [12].

- Diverses applications ont été envisagées pour les fibres de verre recyclées. Les marchés ayant un potentiel intéressant sont ceux des enrobés routiers et des bétons. D'autres débouchés sont à l'étude telle que la réincorporation des fibres dans des matrices thermoplastiques. En effet, d'ores et déjà, les industriels réincorporent des déchets de fibres non ensimées dans le circuit interne, tandis que les fibres ensimées font l'objet d'un recyclage spécifique externe au même titre que les composites. La plupart des industriels ont d'ailleurs créé des sociétés dédiées à la valorisation de leurs déchets ainsi que ceux de leurs clients. C'est le cas de St Gobain avec sa filiale italienne St Gobain-Revetex dont l'objectif est de recycler $90 \%$ des déchets internes. D'un point de vue économique, la réutilisation sélective de la fraction fibreuse semble beaucoup plus prometteuse que le recyclage de la poudre. La fraction de fibres récupérées peut être réutilisée avec succès dans les composites thermodurcissables, en combinaison avec les fibres vierges, sans affecter les propriétés du matériau, dans une proportion pouvant aller jusqu'à $50 \%$ en poids, et avec une économie 
de coût pouvant excéder les $30 \%$. De nombreuses publications et brevets en témoignent (EA1,245 - EP0,473,990 EP0,407,925 - EP0,443,051 - JP6,107,981 - JP2002,167,448 - JP2002,018,845 JP2001,213,982, etc.). Par contre, une réutilisation à $100 \%$ de la fraction fibreuse incorporée dans de nouveaux composites thermodurcissables entraîne une dégradation des propriétés mécaniques. Une solution alternative très prometteuse pourrait être la réutilisation directe de la fraction de fibres récupérées comme renfort de matrices thermoplastiques, telles que le polypropylène et le polyéthylène [8-11].

\section{La valorisation matière des SMC dans les thermoplastiques}

Très peu de brevets ou de publications ont été réalisés dans le domaine de l'incorporation de thermodurcissables ou de composites SMC au sein de thermoplastiques.

Plastic Omnium [16], en collaboration avec MCR/Inoplast, a cependant récemment incorporé en complément à des fibres de verre neuves, des fibres de verre recyclées issues de SMC et provenant de la société MCR, dans une matrice polypropylène, dans le cadre de l'élaboration de pièces techniques sous capot moteur (Renault Laguna). Ce projet a débouché sur une installation industrielle de série. Les propriétés mécaniques obtenues sont acceptables mais bien inférieures à celles de charges ou fibres neuves incorporées dans du polypropylène.

Citons un brevet européen (EP1,508,418) faisant mention de réincorporation de composites SMC simplement déchiquetés dans des thermoplastiques ou autres matrices, ayant en surface des fibres une couverture résiduelle de matière (résine). D'autres brevets (US5,879,601 - FR2,765,123 et US5,569,424) adoptent la même manière de conditionnement par déchiquetage de la matière composite armée en broyat grossier.

Dans un travail réalisé à l'EMA par J.N. Reygrobellet [15], un procédé de broyage/tamisage a été employé pour récupérer les fibres courtes de déchets de composites thermodurcissables et les combiner avec de nouvelles matrices comme agent de renfort. Ce sont uniquement les fractions riches en fibres qui ont été sélectionnées pour être réutilisées. Ces travaux ont montré qu'un procédé de broyage simple et rapide appliqué aux composites polyesters/fibres de verre permettait d'obtenir un matériau broyé acceptable en qualité et en quantité dans les matrices thermoplastiques (PA-6, PP).

Enfin, un brevet japonais (JP5,131,448) fait mention d'une amélioration des propriétés mécaniques de broyats SMC au sein de PP par encapsulation de la charge avec un tensio-actif.

Ces deux dernières références sont à l'origine des objectifs de l'étude. Il s'agit d'augmenter les propriétés mécaniques du compound PP/SMC par amélioration des interfaces en rendant réactif l'ensemble des constituants de SMC (fibre de verre, carbonate de calcium et résine polyester) au moyen d'une fonctionnalisation charge/matrice mais également charge/charge.

\section{Le procédé SyLtec}

\section{But}

L'objectif de l'étude est d'améliorer les propriétés mécaniques de matériaux composites SMC recyclés incorporés dans une matrice polypropylène afin de rendre ce compound compétitif mécaniquement vis-à-vis de composites $\mathrm{PP}$ à fibres de verre vierges. Pour cela, nous proposons un procédé de valorisation de ces charges SMC, dénommé SyLTEC, en quatre étapes.

\section{Implémentation et nomenclature des compounds}

La mise en œuvre est réalisée par extrusion des broyats SMC dans une matrice polypropylène et éventuellement des additifs et agents d'interface selon la phase du procédé.

Les propriétés mécaniques sont mesurées à l'aide d'essais effectués sur des éprouvettes haltères normalisées (norme ISO $527-$ 2 type 1A) et obtenues après injection. La comparaison des propriétés mécaniques, estimées à partir des valeurs du module d'Young (E), de la contrainte au seuil d'écoulement ou à rupture $(\sigma)$ et de la résilience $(R)$, est effectuée à taux de verre constant égale à $20 \%$ en masse du compound final PP/SMC en présence de $5 \%$ de $\mathrm{PP}_{\text {gma }}$ (environ 45 $48 \%$ de charge SMC présent dans le compound suivant phase du procédé Syltec).

La nomenclature utilisée des compounds $\mathrm{PP} / \mathrm{FV}$ neuves et PP/SMC est la suivante : 
$\mathbf{P P 2 0 F V}_{p p}$ : Composite de référence à $20 \%$ en masse de fibres de verre commerciales ensimées polypropylène greffé anhydride maléique.

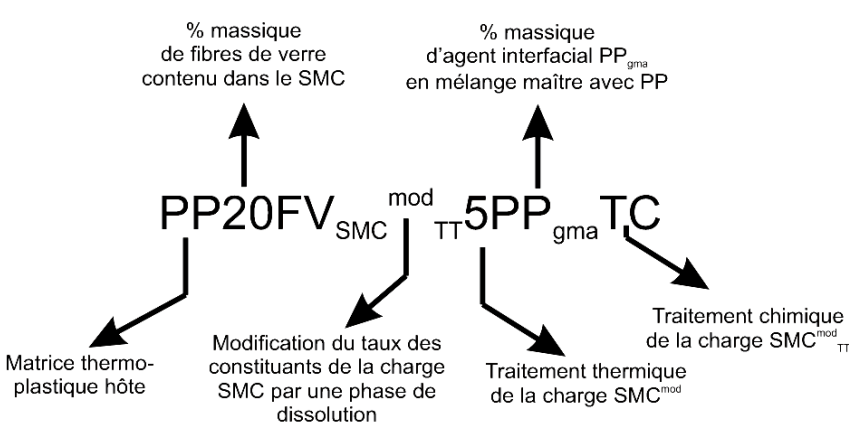

où sont représentés les différents traitements thermiques et chimiques des différentes étapes.

\section{$1^{\text {re }}$ étape : phase de conditionnement (SMC)}

\section{Élaboration de SMC déchiqueté}

La première étape, phase de conditionnement, a eu pour objet une libération des fibres de verre de la résine polyester ainsi qu'une calibration dimensionnelle du broyat de SMC sous forme de matière plus ou moins cotonneuse. Tout d'abord nous nous sommes concentrés sur la phase de libération des fibres de verre par déchiquetage et conditionnement de $4 \mathrm{~kg}(8,82 \mathrm{lbs})$ de déchets SMC. Pour cela, nous avons conditionné les déchets en plaquettes de dimensions moyennes $5 \mathrm{~cm}$ par $5 \mathrm{~cm}$ (1,97 po par 1,97 po) puis utilisé un broyeur déchiqueteur avec grille de passage de matière $\mathrm{d}$ 'ouverture $12 \mathrm{~mm}(0,47 \mathrm{po})$ travaillant par cisaillement de type Rotoplex ${ }^{\circledR}$ (Hosokawa - Alpine) à une vitesse de rotation de $1500 \mathrm{t} / \mathrm{min}^{-1} \mathrm{du}$ rant $25 \mathrm{~min}$. L'étape du procédé ainsi que le broyat SMC obtenu sont décrits figure 3 .

À l'issue de chaque essai, nous avons caractérisé les différentes fractions obtenues en dosant la résine, le carbonate de calcium et en déterminant par analyse d'images, la répartition granulométrique des fibres libérées. Nous avons vu qu'il était possible d'obtenir facilement des broyats d'aspect cotonneux de SMC avec un très bon rendement (de l'ordre de $95 \%$ ) ; cependant, la teneur en fibres restait toujours faible ( $20 \%$ en masse environ), ce qui n'était pas suffisant pour envisager une réincorporation dans les thermoplastiques (propriétés mécaniques insuffisantes vérifiées ultérieurement).
Ajoutons que le déchiquetage permet de préserver la longueur des fibres tout en conservant une faible ségrégation granulométrique. De plus, les observations effectuées par MEB ont montré qu'une partie du carbonate de calcium reste partiellement accrochée à la fibre.

\section{Propriétés mécaniques du PP renforcé par du SMC déchi- queté}

En raison de la faible teneur en FV contenue dans le SMC, il n'est pas possible de mettre en œuvre le compound PP20FV $\mathrm{SMC}_{\text {S }} 5 \mathrm{PP}_{\text {gma }}$, aucune propriété mécanique n'est comparée à celles des fibres de verre neuves contenues dans le PP. Nous présentons cependant les propriétés mécaniques des compounds $\mathrm{PP} / \mathrm{SMC}$ et $\mathrm{PP} / \mathrm{FV}_{\mathrm{pp}}$ à $10 \%$ de $\mathrm{FV}$, soit $\mathrm{PP} 10 \mathrm{FV}_{\mathrm{pp}}$ et PP10FV $\mathrm{FMC}_{\mathrm{SM}} 5 \mathrm{PP}_{\mathrm{gma}}$. Les résultats sont décrits dans le tableau 1 .

Hormis l'augmentation du module E due à la teneur élevée en $\mathrm{CaCO}_{3}$ au sein du $\mathrm{SMC}$, le simple fait de "recycler» le SMC déchiqueté ne peut conduire à des composites acceptables au plan mécanique (faible contrainte et résilience). Un travail de «valorisation » doit être effectué afin d'améliorer les caractéristiques mécaniques du compound $\mathrm{PP} / \mathrm{SMC}$ déchiqueté.

\section{$2^{\mathrm{e}}$ étape : phase de dissolution du SMC}

\section{Élaboration du SMC modifié (SMC ${ }^{\text {mod }}$ )}

La deuxième étape, phase de renforcement matériau, consiste en une étape de dissolution contrôlée du carbonate de calcium selon de la réaction acide-base suivante.

$$
\begin{aligned}
& \mathrm{CaCO}_{3(\mathrm{SMC})}+2 \mathrm{H}_{3} \mathrm{PO}_{4} \Leftrightarrow \\
& \left(\left[\mathrm{Ca}\left(\mathrm{H}_{2} \mathrm{PO}_{4}\right)_{2}\right]_{\text {soluble }}+\mathrm{CO}_{2 \text { diss }}+\mathrm{H}_{2} \mathrm{O} .\right.
\end{aligned}
$$

Cette opération, complétée par un ajustement du taux de fibres de verre, permet d'obtenir une fraction renforçante de $50 \%$ en masse, ce qui correspond à une formulation optimale du compound.

Ce procédé permet une dissolution partielle de près de la moitié de $\mathrm{CaCO}_{3}$ faisant passer la concentration fibreuse de 22 à environ $45,0 \% \pm 1,5$ en masse avec un rendement global de $67 \%$ de la masse initiale en SMC ( $95 \%$ sur la première étape de déchiquetage et $70 \%$ sur l'étape de dissolution partielle). 

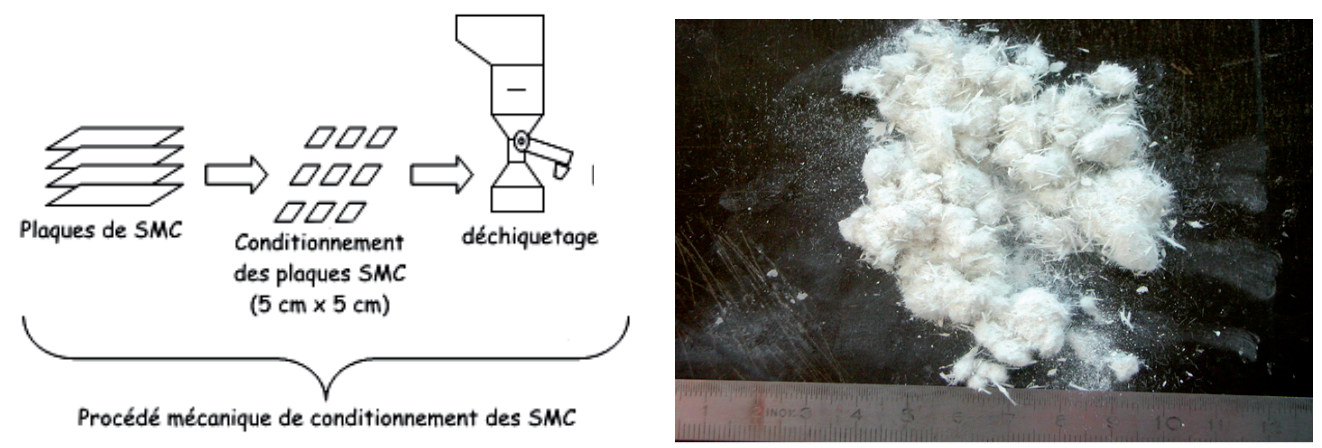

Fig. 3. Procédé de conditionnement et observations du broyat incluant une étape de déchiquetage du déchet SMC.

Fig. 3. Grinding process and study of ground SMC composite material with a first shredding stage.

Tableau 1. Comparaison des caractérisations mécaniques des compounds à matrice PP et à $10 \%$ de FV.

Table 1. Comparison of mechanical properties of composite materials with PP matrix and $10 \%$ w/w E-glass fibres.

\begin{tabular}{|c|c|c|c|}
\hline Composites PP $10 \%$ FV & Module E (MPa) & Contrainte (MPa) & Résilience $\left(\mathrm{kJ} / \mathrm{m}^{2}\right)$ \\
\hline $\begin{array}{l}\mathrm{PP}_{0 \mathrm{FV}} \\
\text { (référence) }\end{array}$ & $3260 \pm 50$ & $46,4 \pm 0,5$ & $25 \pm 1$ \\
\hline $\begin{array}{l}\text { PP10FV } \\
\text { (1 }{ }^{\text {re }} \text { étape du procédé SYLTEC) }\end{array}$ & $4010 \pm 30$ & $27,5 \pm 0,4$ & $9 \pm 1$ \\
\hline
\end{tabular}

La solution d'attaque acide résultant du procédé de dissolution est entièrement recyclée par réajustement du $\mathrm{pH}$ au $\mathrm{pH}$ initial.

La réaction de dissolution partielle $d u$ carbonate de calcium dégage du dioxyde de carbone qui reste piégé dans la solution en raison d'un $\mathrm{pH}$ supérieur à 1,46 (limite

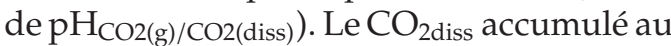
cours des cycles de dissolution partielle successifs peut être éliminé par distillation.

\section{Propriétés mécaniques du SMCmod dans le PP}

Nous présentons les propriétés mécaniques des compounds PP/SMC mod à $10 \%$ de FV, soit PP10FV $\mathrm{SMC}_{\mathrm{SM}} 5 \mathrm{PP}_{\mathrm{gma}}$ afin de faire le lien entre la première et la deuxième étape $\mathrm{du}$ procédé. Les résultats sont décrits dans le tableau 2.

Cependant, compte tenu de la teneur en fibres de verre dans le SMC ${ }^{\text {mod }}$, il est possible de mettre en œuvre des compounds à $20 \%$ en FV. Nous présentons par conséquent les compounds PP20FV $\mathrm{pp}_{\mathrm{p}}$ et PP20FV $\mathrm{SMC}_{\mathrm{SM}}^{\mathrm{mod}} 5 \mathrm{PP}_{\text {gma }}$ dans le tableau 3.

Nous obtenons une augmentation des propriétés mécaniques dues principalement à l'augmentation de la teneur en fibres de verre.

\section{$3^{e}$ étape : phase de traitement thermique de SMC ${ }^{\text {mod }}$}

\section{Élaboration de SMC ${ }^{\text {mod }}$ traité thermiquement (SMC mod)}

La troisième étape, phase de traitement thermique, crée, par fonctionnalisation du polyester, une adhésion ionique supplémentaire, charge/charge (carbonate de calcium - anhydride du polyester thermiquement activé) en plus de l'interaction déjà présente charge/matrice (carbonate de calcium - anhydride de l'agent interfacial $\mathrm{PP}_{\text {gma }}$ ). Cela permet un renforcement conséquent $\mathrm{du}$ composite SMC/matrice PP.

Tout l'intérêt de ce traitement, cœur de l'étude, est d'optimiser au mieux le temps et la durée de traitement thermique (TT) afin de générer la chimie adéquate nécessaire à l'obtention de polyesters fonctionnalisés.

\section{Propriétés mécaniques du SMC mod dans le PP}

Nous présentons les propriétés mécaniques des compounds $\mathrm{PP} / \mathrm{SMC}_{\mathrm{TT}}^{\text {mod }}$ à $20 \%$ de $\mathrm{FV}$, soit PP20FV $\mathrm{SMC}_{\mathrm{SM}}^{\mathrm{TT}} 5 \mathrm{PP}_{\mathrm{gma}}$. Les résultats sont décrits dans le tableau 4.

Si l'on compare les valeurs de module élastique pour un taux de fibres de verre de $20 \%$, nous observons des évolutions de 
Tableau 2. Comparaison des caractérisations mécaniques des compounds à matrice PP et à $10 \%$ de FV.

Table 2. Comparison of mechanical properties of composite materials with PP matrix and $10 \%$ w/w E-glass fibres.

\begin{tabular}{|c|c|c|c|}
\hline Composites PP $10 \%$ FV & Module E (MPa) & Contrainte (MPa) & Résilience $\left(\mathrm{kJ} / \mathrm{m}^{2}\right)$ \\
\hline $\begin{array}{l}\mathrm{PP} 10 \mathrm{FV} \\
\text { (référence) }\end{array}$ & $3260 \pm 50$ & $46,4 \pm 0,5$ & $25 \pm 1$ \\
\hline 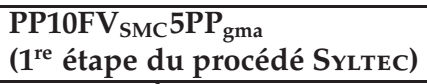 & $4010 \pm 30$ & $27,5 \pm 0,4$ & $9 \pm 1$ \\
\hline $\begin{array}{l}\text { PP10FV } \\
\left(2^{\mathrm{e}} \text { étac } 5 \text { é }\right)\end{array}$ & $2750 \pm 40$ & $36,5 \pm 0,2$ & $14 \pm 1$ \\
\hline
\end{tabular}

Tableau 3. Comparaison des caractérisations mécaniques des compounds à matrice PP et à $20 \%$ de FV.

Table 3. Comparison of mechanical properties of composite materials with PP matrix and $20 \% \mathrm{w} / \mathrm{w}$ E-glass fibres.

\begin{tabular}{|c|c|c|c|}
\hline Composites PP $20 \%$ FV & Module E (MPa) & Contrainte (MPa) & Résilience $\left(\mathrm{kJ} / \mathrm{m}^{2}\right)$ \\
\hline $\begin{array}{l}\mathrm{PP20FV}_{\mathrm{pp}} \\
\text { (référence) }\end{array}$ & $5070 \pm 20$ & $57,6 \pm 0,2$ & $18 \pm 1$ \\
\hline $\begin{array}{l}\text { PP20FV } \\
\left(2^{\mathrm{e} o d} \text { étape }\right)\end{array}$ & $3750 \pm 30$ & $38,4 \pm 0,5$ & $11 \pm 1$ \\
\hline
\end{tabular}

Tableau 4. Caractérisations mécaniques des compounds à matrice PP et à $20 \%$ de FV.

Table 4. Comparison of mechanical properties off composite materials with PP matrix and $20 \% \mathrm{w} / \mathrm{w}$ E-glass fibres.

\begin{tabular}{|c|c|c|c|}
\hline Composites PP $20 \%$ FV & Module E (MPa) & Contrainte (MPa) & Résilience $\left(\mathrm{kJ} / \mathrm{m}^{2}\right)$ \\
\hline $\begin{array}{l}\mathrm{PP20FV}_{\mathrm{pp}} \\
\text { (référence) }\end{array}$ & $5070 \pm 20$ & $57,6 \pm 0,2$ & $18 \pm 1$ \\
\hline $\begin{array}{l}\text { PP20FV } \\
\left(2^{\mathrm{e}} \text { étac }\right. \\
\text { étaP }\end{array}$ & $3750 \pm 30$ & $38,4 \pm 0,5$ & $11 \pm 1$ \\
\hline $\begin{array}{l}\text { PP20FV } \\
\text { (3 étac TT } 5 \text { étape) }\end{array}$ & $5100 \pm 50$ & $51 \pm 0,3$ & $14 \pm 1$ \\
\hline
\end{tabular}

propriétés très différentes entre les échantillons traités thermiquement et ceux qui ne le sont pas :

1. Pour les échantillons non traités thermiquement $\left(\mathrm{PP} \times \mathrm{FV}_{\mathrm{SMC}}^{\mathrm{mod}}\right.$ et $\left.\mathrm{PP} \times \mathrm{FV}_{\mathrm{SMC}}^{\mathrm{mod}} 5 \mathrm{PP}_{\text {gma }}\right)$, les composites sans $\mathrm{PP}_{\text {gma }}$ présentent un module élastique plus élevé que les composites avec $5 \% \mathrm{PP}_{\text {gma, }}$, ce qui s'explique par le fait que le $\mathrm{PP}_{\text {gma }}$ peut plastifier la matrice PP.

2. Pour les échantillons traités thermiquement $\quad\left(\mathrm{PP} \times \mathrm{FV}_{\mathrm{SMC}}^{\mathrm{mod}} \mathrm{TT}\right.$ et PP $\times F_{\text {SMC }}^{\text {mod }}$ TT5PP gma), nous observons la tendance inverse : les composites sans $\mathrm{PP}_{\text {gma }}$ ont un module élastique plus faible que ceux avec $\mathrm{PP}_{\text {gma, }}$, ce qui peut s'expliquer, d'une part par une réaction du $\mathrm{PP}_{\text {gma }}$ avec les charges ( $\mathrm{d}$ 'où une meilleure adhésion charge/matrice et un effet de renforcement important qui contrebalance la plastification de la matrice $\mathrm{PP}$ par le $\mathrm{PP}_{\text {gma }}$ ), et d'autre part, il est probable que le $\mathrm{PP}_{\text {gma }}$ introduit soit concentré à l'interface charge/matrice et donc présent en plus faible quantité dans la matrice PP, qui se trouve alors moins plastifiée.
Par conséquent, la réalisation d'un traitement thermique permet d'améliorer l'effet $\mathrm{du} \mathrm{PP}_{\text {gma }}$ sur la contrainte au seuil et la résilience et ceci, sans effet négatif sur le module élastique.

Le traitement thermique du polyester permet donc d'imaginer un produit SMC recyclé non seulement compétitif vis-à-vis de produits commerciaux de même nature mais également vis-à-vis de fibres de verre E ensimées. Cependant, les propriétés mécaniques en résilience demeurent en retrait vis-à-vis de celles des compounds PP/FV $\mathrm{PP}$.

\section{$4^{e}$ étape : phase de traitement chimique de SMC mod}

\section{Élaboration du SMC mod traité thermiquement (SMC mod TC)}

La dernière étape, phase de traitement chimique, apporte une fonctionnalisation chimique entre la charge (SMC modifiée traitées thermiquement) et la matrice active $\left(\mathrm{PP} / \mathrm{PP}_{\text {gma }}\right)$, par l'intermédiaire d'un agent polyfonctionnel type amine. La réaction permet ainsi la création d'une véritable liaison chimique entre le renfort et la charge. Cette 
étape n'est pas nécessaire dans le cas d'une matrice polaire comme le PA-6.

L'agent chimique interfacial utilisé, de formulation générale $\mathrm{M}-\left(\mathrm{CH}_{2}\right)_{\mathrm{x}}-\mathrm{M}(\mathrm{M}$ : tête polaire et $\mathrm{x}$ : nombre de liaisons aliphatiques $\mathrm{CH}_{2}$ au sein du composé), est un composé multipolaire qui permet :

$1^{\circ}$ ) une réaction chimique des anhydrides du polyester fonctionnalisé et de l'agent,

$2^{\circ}$ ) une réaction chimique des anhydrides du PP/PP gma et de la charge SMC contenant le polyester fonctionnalisé thermiquement. Cette opération s'effectue par extrusion réactive.

Comme pour l'étape de traitement thermique, il est indispensable de déterminer précisément les conditions optimales de fonctionnalisation chimique en temps et température de réaction (optimisation réalisée par plan d'expérience, puis par réaction par DSC et analyse par IRTF).

$\mathrm{Au}$ final, le protocole expérimental requis est défini comme suit :

1. prise de $\mathrm{y} \mathrm{kg}$ de déchet $\mathrm{SMC}_{\mathrm{TT}}^{\mathrm{mod}}$,

2. incorporation du déchet dans $\mathrm{y} / 10 \mathrm{~L}$ d'eau/éthanol (50/50 v/v) contenant y ml d'agent interfacial,

3. mise à l'étuve du déchet fonctionnalisé suivant des conditions de traitement optimisées en temps et en température nécessaire à l'avancement maximal de la réaction de fonctionnalisation entre l'agent chimique interfacial et la charge $\mathrm{SMC}_{\mathrm{TT}}^{\mathrm{mod}}$,

4. obtention du $\mathrm{SMC}_{\mathrm{TT}}^{\text {mod }} \mathrm{TC}$ sous forme terreuse (facilement mis en œuvre par extrusion).

\section{Propriétés mécaniques du SMC modTC dans le PP}

Les systèmes composites obtenus avec des charges de SMC ayant subi les 4 phases du procédé (déchiquetage / attaque acide du carbonate de $\mathrm{Ca} /$ traitement thermique / imidification) ont été comparés aux composites précédemment élaborés tout au long du projet, excepté le composite PP20FV $\mathrm{FMC}_{\text {SM }}$. Le taux final en fibres de verre est toujours fixé à $20 \%$. L'ensemble des résultats est décrit dans le tableau 5.

Une légère diminution de la contrainte et du module est observée sur le composite PP20FV SMC $_{\text {TT }} 5$ PP $_{\text {gma }}$ en présence d'agent interfacial.

Toutefois, ce composite présente une résilience nettement améliorée mise en évidence au sein de ce composite. La création d'une liaison chimique à l'interface permet d'interpréter ce résultat.

Ajoutons qu'en faisant varier la longueur $x$ de la chaîne de l'agent interfacial, il est possible d'obtenir des comportements rhéologiques mécaniques en fonction des applications visées (élastique, viscoélastique, viscoplastique, etc.).

$\mathrm{Au}$ bilan, ce procédé de valorisation de charges SMC recyclés incorporées dans du polypropylène conduit à des propriétés mécaniques sensiblement comparables à celles de fibres neuves incorporées dans la même matrice.

Il est à rappeler que ce procédé est transposable à des matrices polaires (PA, etc.) avec suppression de la quatrième étape.

\section{Transposition à l'échelle pilote}

Le procédé Syltec offre une voie technologiquement performante et économiquement viable de diminuer de manière conséquente la mise en décharge de pièces composites SMC (capots de voiture, custodes, hayons, pare-chocs, cadre de pièces techniques sous capot moteur ou en tant que renfort comme les passages de roues, etc.) en les valorisant sous la forme d'un broyat concurrençant les fibres de verre comme renfort dans certains matériaux composites à matrice thermoplastique.

Une installation pilote a vu le jour suite à ces travaux en partenariat avec un industriel Rhône-alpin du recyclage et un dépôt de brevet est en cours. Enfin, ajoutons que la stratégie développée dans ce procédé peut être transposée à d'autres composites à matrice thermodurcissable.

\section{Conclusion}

La gestion des déchets plastiques et composites, comme celle des autres déchets, est devenue incontournable. On ne peut admettre que des objets ayant demandé de l'énergie et beaucoup d'efforts soient abandonnés sans tentative de valorisation. Cette philosophie a été le thème de cette étude appliquée à des matériaux composites à matrices thermodurcissables et fibres de verre.

L'objectif ainsi fixé était, par le biais d'un procédé de recyclage et de valorisation des composites SMC dans des thermoplastiques apolaires (PP), dénommé Syltec, d'obtenir des propriétés mécaniques susceptibles de concurrencer celles des composites à fibres 
Tableau 5. Comparaison des caractérisations mécaniques des compounds à matrice PP et à $20 \%$ de FV.

Table 5. Comparison of mechanical properties of composite materials with PP matrix and $20 \% \mathrm{w} / \mathrm{w}$ E-glass fibres.

\begin{tabular}{|c|c|c|c|}
\hline Composites PP $20 \%$ FV & Module E (MPa) & Contrainte (MPa) & Résilience $\left(\mathrm{kJ} / \mathrm{m}^{2}\right)$ \\
\hline $\begin{array}{l}\text { PP20FV } \\
\text { (référence) }\end{array}$ & $5070 \pm 20$ & $57,6 \pm 0,2$ & $18 \pm 1$ \\
\hline $\begin{array}{l}\text { PP20FV } \\
\text { (2e étape) }\end{array}$ & $3750 \pm 30$ & $38,4 \pm 0,5$ & $11 \pm 1$ \\
\hline $\begin{array}{l}\text { PP20FV } \\
\text { (3) étac TT } 5 \text { eP }_{\text {gma }}^{\text {mod }}\end{array}$ & $5100 \pm 50$ & $51,0 \pm 0,3$ & $14 \pm 1$ \\
\hline $\begin{array}{l}\text { PP20FV }_{\text {SMC }}^{\text {mod }} \text { TC }^{\text {TCP }} \\
\text { (4ma étape) }\end{array}$ & $4900 \pm 60$ & $48,6 \pm 0,6$ & $22 \pm 1$ \\
\hline
\end{tabular}

vierges tout en réincorporant l'intégralité de la charge dans la matrice. Pour cela, une bonne maîtrise des performances mécaniques des matériaux composites a nécessité la connaissance aussi précise que possible de l'interphase et interface entre les constituants du recyclât SMC (carbonate de calcium, fibres de verre et résine polyester insaturée) et entre cette charge et la matrice thermoplastique hôte. Aussi, nous nous sommes concentrés sur la fonctionnalisation de l'ensemble des constituants du composite final, avant et après incorporation de la charge SMC traitée, par le biais du procédé Syltec développé au cours de ces travaux.

Le procédé original de recyclage des matériaux SMC par valorisation de ceux-ci dans des matrices thermoplastiques a conduit à l'entière fonctionnalisation de la charge SMC par un processus en quatre étapes, pour des matrices thermoplastiques apolaires comme le PP, et en trois étapes dans le cas de matrices thermoplastiques polaires comme le PA-6.

Dans chaque étape, nous avons :

- déterminé très précisément le rôle joué par les constituants de la charge SMC sur les propriétés physico-chimiques du composite final,

- isolé et fonctionnalisé le constituant susceptible de contribuer à l'amélioration de ces propriétés par l'intermédiaire de traitements appropriés,

- évalué les interactions entre constituants de la charge et entre la charge et la matrice, par l'intermédiaire de plans d'expériences, de manière à optimiser au mieux les interactions de chaque élément du matériau composite final,

- gardé comme objectif, une méthode de valorisation simple, peu coûteuse en temps et en énergie.
Aussi, à partir de ces différentes évaluations et de la connaissance physico-chimique des différentes interactions entre élément, nous avons pu proposer une schématisation et une modélisation des mécanismes réactionnels d'interface engagés pour chaque élément du composite final dans chacune des phases du procédé de valorisation du SMC.

\section{Références}

[1] B. Mellian, Advanced Mater. Process 2 (1997) $47-48$

[2] C.L. Smith, F.J. Good, Plastics Eng. (1996) 2728

[3] CSTB Magazine 90 (1995) 16-20

[4] GPIC, Avril 2000

[5] N. Rizzi, Agence Rhône-Alpes pour la Maîtrise des Matériaux, Sept. 2002, France

[6] J.C. Duffés, Rev. des ing. de l'auto, Mai 2001

[7] G. Marsh, Reinforced plastics, June 2001

[8] R.U. Ayres, Ecological Economics 48 (2004) $425-438$

[9] La valorisation des déchets en composites thermodurcissables, Journée Rhône-Alpes des composites, Oct. 2003

[10] J. Takahashi, Composites 35 (1999) 58-61

[11] C.E. Kouparitsas, C.N. Kartalis, P.C. Varelidis, C.J. Tsenoglou, C.D. Papaspyrides, Polym. Comp. 23 (2002) 682-689

[12] G. Marsh, Reinforced plastics, June 2001

[13] Recycling takes off at MCR, Composites International 48 (2001) 27-31

[14] A. Marion, Mecelec Composites et Recyclage, Composites 35 (1999) 49-51

[15] J.N. Reygrobellet, Recyclage de composites fibres de verre - polyester insaturé - carbonate de calcium par réincorporation dans des matrices thermoplastiques, Thèse de Doctorat, Université Montpellier II, 2000

[16] F. Viot, Valorisation de thermodurcissables dans des thermoplastiques, Plastic Omnium, Séminaire international sur les solutions concrètes pour le recyclage des matériaux composites, 28 et 29 juin 2005 